\title{
Heavy Metal Bioaccumulation, Growth Characteristics, and Yield of Pisum sativum L. Grown in Agricultural Soil-Sewage Sludge Mixtures
}

\author{
Ebrahem M. Eid ${ }^{1,2} \oplus$, Ahmed F. El-Bebany ${ }^{3, *}$, Mostafa A. Taher ${ }^{1,4}$, Sulaiman A. Alrumman ${ }^{1}{ }^{1}$, \\ Tarek M. Galal ${ }^{5,6}$, Kamal H. Shaltout ${ }^{7}$, Nasser A. Sewelam ${ }^{7}$ and Mohamed T. Ahmed ${ }^{1}$ \\ 1 Biology Department, College of Science, King Khalid University, Abha 61321, Saudi Arabia; \\ ebrahem.eid@sci.kfs.edu.eg (E.M.E.); mostafataherahm@yahoo.com (M.A.T.); \\ salrumman@kku.edu.sa (S.A.A.); mtahmed@kku.edu.sa (M.T.A.) \\ 2 Botany Department, Faculty of Science, Kafrelsheikh University, Kafr El-Sheikh 33516, Egypt \\ 3 Plant Pathology Department, Faculty of Agriculture, Alexandria University, El-Shatby, \\ Alexandria 21545, Egypt \\ 4 Botany Department, Faculty of Science, Aswan University, Aswan 81528, Egypt \\ 5 Botany and Microbiology Department, Faculty of Science, Helwan University, Cairo 11795, Egypt; \\ tarek_abosarea@science.helwan.edu.eg \\ 6 Biology Deprtment, College of Science, Taif University, Taif 21974, Saudi Arabia \\ 7 Botany Department, Faculty of Science, Tanta University, Tanta 31527, Egypt; \\ kamal.shaltout@science.tanta.edu.eg (K.H.S.); sewelam@science.tanta.edu.eg (N.A.S.) \\ * Correspondence: ahmed.elbebany@alexu.edu.eg or aelbebany@yahoo.com; Tel.: +20-1123158526
}

Received: 31 August 2020; Accepted: 30 September 2020; Published: 1 October 2020

\begin{abstract}
The application of sewage sludge (SS) in agriculture is an alternative disposal method for wastewater recycling and soil fertilization. This study evaluated heavy metal bioaccumulation, growth, and yield of Pisum sativum (pea) grown in agricultural soil amended with SS at rates of 0, 10, 20,30 , and $40 \mathrm{~g} / \mathrm{kg}$. The results show that root, shoot, pod length, biomass, and number of leaves and pods increased with SS amendments of 10 and $20 \mathrm{~g} / \mathrm{kg}$, while rates declined at 30 and $40 \mathrm{~g} / \mathrm{kg}$. SS had greater salinity and organic content than the soil. Heavy metals in the postharvest soil samples increased for all SS application rates except $\mathrm{Fe}$ and Mo. The significant increase in $\mathrm{Cd}$ content started at the lowest amendment rate $10 \mathrm{~g} / \mathrm{kg}$; for $\mathrm{Co}, \mathrm{Mn}$, and $\mathrm{Pb}$, the significant increase was detected at the highest amendment rate $(40 \mathrm{~g} / \mathrm{kg})$. Generally, all heavy metals increased significantly in portions of $P$. sativum except $\mathrm{Cd}$ in the shoot. At an amendment rate of $10 \mathrm{~g} / \mathrm{kg}$, Co in the shoot and root, $\mathrm{Cr}$ in the fruit, $\mathrm{Cu}$ in the root, $\mathrm{Fe}$ in the fruit, $\mathrm{Mn}$ in the shoot and fruit, $\mathrm{Mo}$ in the fruit, $\mathrm{Pb}$ in the shoot, and $\mathrm{Zn}$ in the fruit were elevated significantly. In contrast, the concentrations of $\mathrm{Cd}$ in the fruit, $\mathrm{Cr}$ in the root, $\mathrm{Cu}$ in the shoot, $\mathrm{Fe}$ in the shoot and root, $\mathrm{Ni}$ in the fruit and root, $\mathrm{Pb}$ in the fruit and root, and $\mathrm{Zn}$ in the root significantly increased only at the highest rate of $40 \mathrm{~g} / \mathrm{kg}$. The highest regression $R^{2}$ was 0.927 for $\mathrm{Mn}$ in pods and the lowest was 0.154 for $\mathrm{Cd}$ in shoots. Bioaccumulation and translocation factors were $>1$ for $\mathrm{Mo}$ and the bioaccumulation of $\mathrm{Pb}$ was $>1$. SS could be used for pea fertilization but only at rates below $20 \mathrm{~g} / \mathrm{kg}$ to avoid environmental and health hazards.
\end{abstract}

Keywords: pea; soil amendment; accumulation; metal translocation; fertilization

\section{Introduction}

The rapid expansion of population in several countries increases pressure on the agriculture to produce more food. Low soil fertility in Saudi Arabia and several surrounding countries plays a role in the decline of crop production. On the edges of towns and cities, there are usually some rural areas 
that produce daily vegetables. Sewage sludge (SS) provides agricultural soils with organic matter and several macro- and micronutrients which are used as fertilization materials [1], and its amendment, inducing alteration of soil $\mathrm{pH}$, affects the bioavailability of several elements. For these reasons, the application of SS is considered a low-cost waste disposal method reducing crop production costs [2].

Numerous investigations have studied the influence of municipal SS application on many crops in terms of their growth, productivity, and metal uptake. Investigated crops include trees such as olive [3], field crops such as alfalfa [4], rice [5], corn [6], wheat [7-10], barley [11,12], cotton [13], and broad bean [14], vegetable crops such as French bean [15], cucumber [16,17], cherry tomato [18], sweet pepper [19], cabbage and broccoli [20], as well as spinach [21-23]. However, variations in SS source and composition, agricultural soil type, and environmental conditions require more specific estimates for different cropping systems [24]. Heavy metal content in SS is a limiting factor for SS application due to the toxic effects of these metals on plants. Heavy metals phytotoxicity includes alteration of pigment biosynthesis, photosynthesis process, inactivation of enzymes, nutrients assimilation and plant hormonal balance [25-27]. Monitoring heavy metals in plants growing in soils amended with SS is an important issue as the accumulation of heavy metals may contaminate the food chain through entering animal and human bodies, causing severe health disorders [25].

Pea (Pisum sativum L.) is a legume plant and member of the family Fabaceae. It is an important pulse crop that provides human and animals with nutritional values. P. sativum is considered a cheap source of protein (average of $20-22 \%$ protein per seed) in developing countries [28]. Several studies demonstrated that sewage irrigation improved the nodulation on the root system of $P$. sativum but suppressed the colonization of mycorrhiza [29]. Germination of P. sativum and other legume seeds was negatively affected by industrial wastewater applications [30]. Previous experiments, considering SS's impact on another legume, Faba sativa, indicated a dose-dependent improvement in growth and yield starting with increasing the biomass and yield followed by decline at high concentrations of SS amendments [14]. Interestingly, SS compost has been reported as suppressing the damping-off disease (caused by Rhizoctonia solani and Pythium ultimum) in P. sativum [31]; however, the effect of SS compost differs according to the crop and the associated pathogen type [32].

The objectives of this research were to assess the impact of SS amendments to agricultural soil on the growth and biomass of pea, and to determine the bioaccumulation and transformation of heavy metals in different portions of pea plants.

\section{Results and Discussion}

\subsection{Chemical Analyses of Sewage Sludge and Agricultural Soil}

The data in Table 1 show the chemical analysis of both SS and cultivated fields' soil before mixing. SS had higher salinity than the agricultural soil, and had an organic matter content approximately 65 times more than that of the soil. The agricultural soil was more alkaline than the SS (which was neutral, $\mathrm{pH}$ 6.98). High organic matter content in the SS resembled previous analyses on other plants from the same municipal station [14]. The permissible and average normal limits of heavy metals in SS and agricultural soil are shown in Table 1. Heavy metal analysis showed that SS had higher values of $\mathrm{Cr}, \mathrm{Cu}, \mathrm{Ni}, \mathrm{Pb}$, and $\mathrm{Zn}$ than the agricultural soil. However, $\mathrm{Fe}, \mathrm{Mn}, \mathrm{Cd}$, and Co content was higher in the cultivated soil. Although all the measured heavy metals in SS were lower than the permissible limits [33], the additive effect of using SS in agricultural soil should be monitored in order to keep the content of these metals within the average normal limits [34].

\subsection{Plant Growth Measurements}

The effects of different SS amendment rates on the morphology and morphometric measurements of $P$. sativum are illustrated in Figures 1 and 2. Germination of $P$. sativum seeds was negatively affected at all SS amendment rates. The effects on the germination percentage appear to be related to heavy metal concentration in SS. $\mathrm{Cr}$ and $\mathrm{Cu}$ have been reported as inhibitors of P. sativum germination, and 
they also suppressed the germination and seedling growth of wheat $[25,35,36]$. However, root, shoot, and pod length, number of leaves and pods, and absolute growth rate of the plant gradually increased at low rates of SS amendments, followed by decline at amendment rates of 30 and $40 \mathrm{~g} / \mathrm{kg}$ (Figure 2). The same trend was observed for root, shoot, pod, above-ground and total biomasses of P. sativum (Figure 3). The enhancement of $P$. sativum growth and yield may be due to the organic materials and micronutrients in the low levels of SS amendments [28]. On the contrary, using SS compost did not increase the pea yield but did increase the yield of the monocot plants triticale and maize [1]. The reduction in pea growth at high rates of SS amendments could be due to nutrient deficiency. The antagonistic or synergistic effect of heavy metals on uptake of elements was reported in many studies; Cd inhibited the absorption and accumulation of K, Ca and Fe [37,38]. It has been reported that similarities of heavy metals with nutrients cations result in competition for absorption by root cells $[25,26]$. At the high rates of SS amendments, heavy metals enter into the cells of plant roots and may affect the primary metabolic pathways through direct interaction with sulfhydryl groups of proteins, which consequently reduces plant growth [39]. The generation of reactive oxygen species in plant cells as a response to heavy metals may cause damage to macromolecules such as proteins and lipids, which results in the reduction in plant growth [27]. In addition, the decline in plant biomass may refer to the SS salinity and/or inhibition of growth hormones biosynthesis in plants grown in soil with a high content of SS [40].

Table 1. Selected chemical properties of sewage sludge and agricultural soil used in the pot experiment (means \pm standard error, $n=3$ ).

\begin{tabular}{ccccc}
\hline & \multicolumn{2}{c}{ Sewage Sludge } & \multicolumn{2}{c}{ Agricultural Soil } \\
\cline { 2 - 5 } Properties & Measured Values & Permissible Limits * & Measured Values & Average Normal Limits ** \\
\hline Salinity $(\mathrm{mS} / \mathrm{cm})$ & $1.39 \pm 0.10$ & NA & $0.07 \pm 0.00$ & NA \\
pH & $6.98 \pm 0.02$ & NA & $8.68 \pm 0.02$ & NA \\
Organic matter $(\%)$ & $65.0 \pm 0.9$ & NA & $0.9 \pm 0.2$ & NA \\
Cd $(\mathrm{mg} / \mathrm{kg})$ & $1.17 \pm 0.08$ & $20.0-40.0$ & $2.91 \pm 0.05$ & 3.0 \\
$\mathrm{Co}(\mathrm{mg} / \mathrm{kg})$ & $25.86 \pm 1.31$ & - & $35.49 \pm 1.13$ & 35.0 \\
$\mathrm{Cr}(\mathrm{mg} / \mathrm{kg})$ & $176.18 \pm 1.94$ & 900.0 & $134.34 \pm 0.66$ & 125.0 \\
$\mathrm{Cu}(\mathrm{mg} / \mathrm{kg})$ & $162.56 \pm 2.32$ & $1000.0-1750.0$ & $15.01 \pm 0.57$ & 105.0 \\
$\mathrm{Fe}(\mathrm{mg} / \mathrm{g})$ & $24.41 \pm 0.52$ & - & $42.37 \pm 0.53$ & 39.2 \\
$\mathrm{Mn}(\mathrm{mg} / \mathrm{kg})$ & $560.70 \pm 9.81$ & - & $677.27 \pm 3.23$ & 775.0 \\
$\mathrm{Mo}(\mathrm{mg} / \mathrm{kg})$ & $0.91 \pm 0.04$ & - & $1.06 \pm 0.01$ & 7.0 \\
$\mathrm{Ni}(\mathrm{mg} / \mathrm{kg})$ & $138.73 \pm 3.71$ & $300.0-400.0$ & $68.09 \pm 3.70$ & 40.0 \\
$\mathrm{~Pb}(\mathrm{mg} / \mathrm{kg})$ & $671.11 \pm 6.22$ & $750.0-1200.0$ & $3.51 \pm 0.39$ & 160.0 \\
$\mathrm{Zn}(\mathrm{mg} / \mathrm{kg})$ & $667.62 \pm 13.44$ & $2500.0-4000.0$ & $77.18 \pm 1.94$ & 200.0 \\
\hline
\end{tabular}

*: He et al. (2005), **: Kabata-Pendias (2011), NA: not applicable.

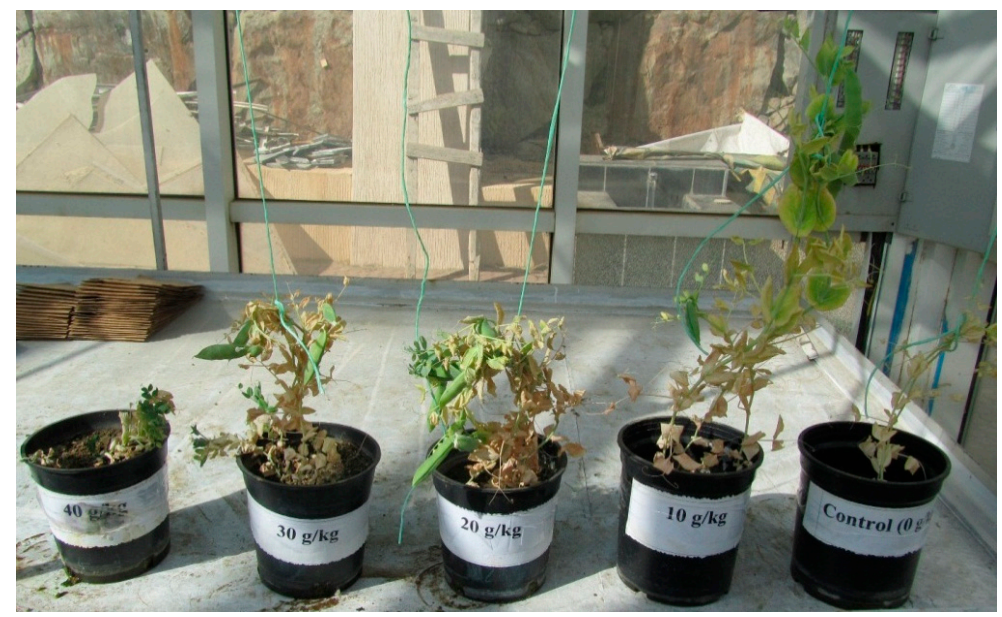

Figure 1. A demonstration of five pots (replicates) represents the Pisum sativum plants grown in sewage sludge-soil with amendment rates of 0 (control), 10, 20, 30 and $40 \mathrm{~g} / \mathrm{kg}$. 

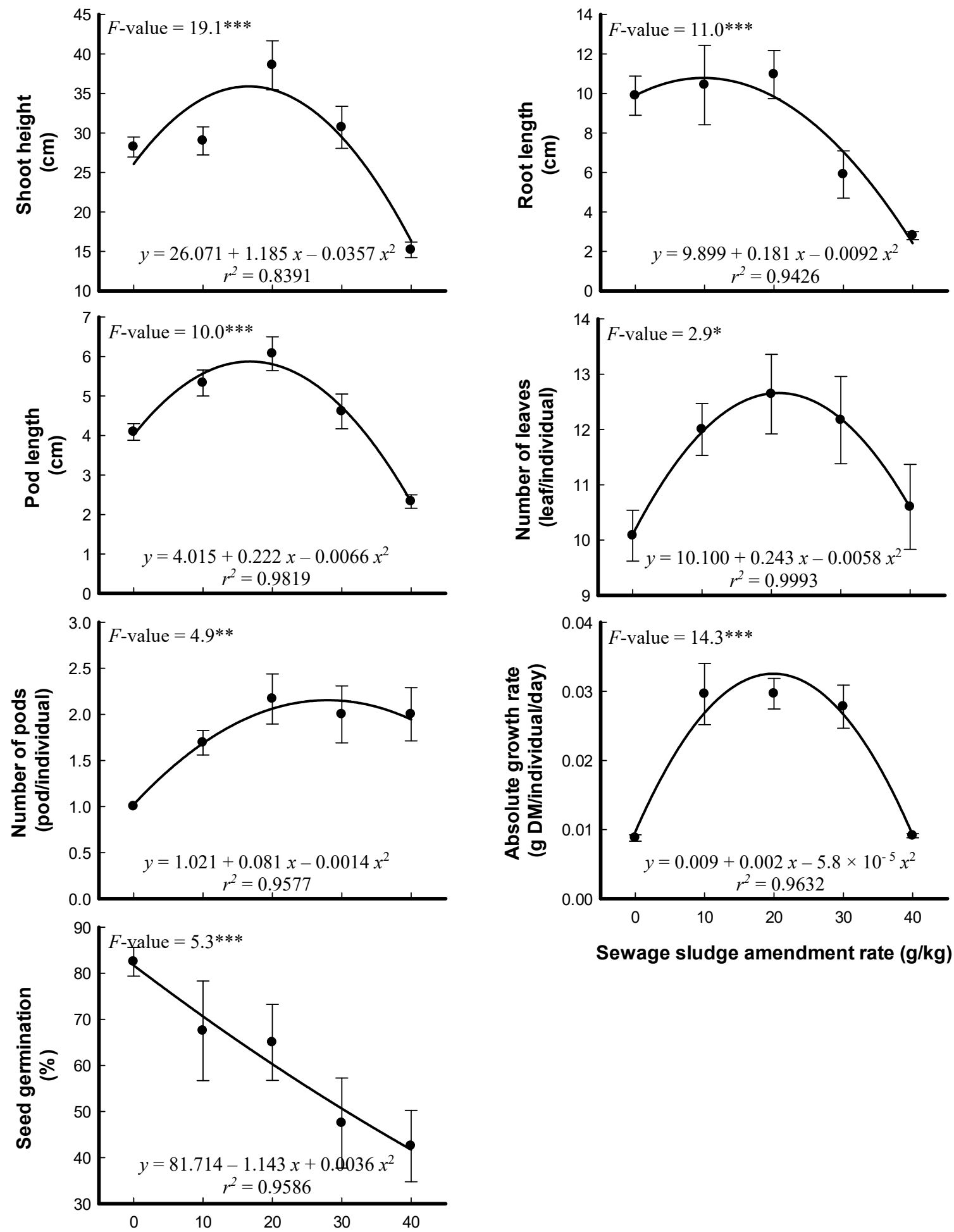

Sewage sludge amendment rate $(\mathrm{g} / \mathrm{kg})$

Sewage sludge amendment rate $(\mathrm{g} / \mathrm{kg})$

Figure 2. Effects of different sewage sludge amendment rates on the morphometric parameters of Pisum sativum that had been grown for 57 days (means \pm standard error, $n=6$ ). The $F$-values represent one-way analysis of variance (ANOVA) and a degree of freedom $(d f)=4$. $^{*}: p<0.05,{ }^{* *}: p<0.01,{ }^{* * *}: p$ $<0.001$. 

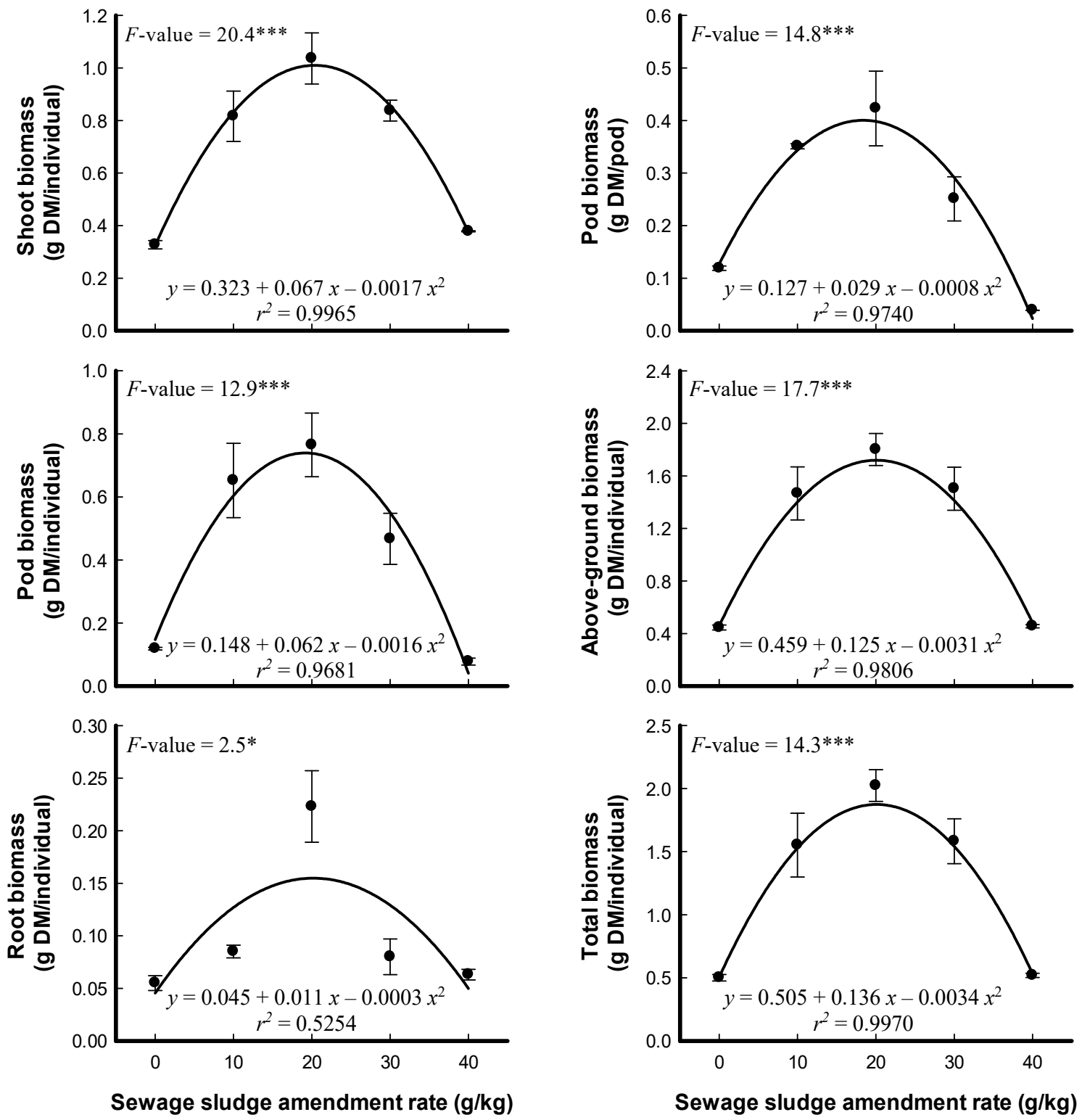

Figure 3. Effects of different sewage sludge amendment rates on the biomass of Pisum sativum that had been grown for 57 days (means \pm standard error, $n=6$ ). $F$-values represent a one-way ANOVA and degrees of freedom $(d f)=4 .^{*}: p<0.05,{ }^{* * *}: p<0.001$.

\subsection{Postharvest Chemical and Heavy Metal Analyses of Soil and Pea Portions}

The chemical analysis of samples collected from soil for all SS treatments after P. sativum harvesting is shown in Table 2. A significant increase in salinity was detected at amendment rates of 30 and $40 \mathrm{~g} / \mathrm{kg}$ and the organic matter was increased significantly under all amendment rates, whereas pH significantly decreased until reaching 7.09 at an amendment rate $40 \mathrm{~g} / \mathrm{kg}$. Heavy metals in the postharvest soil samples increased for all SS application rates except Fe and Mo. The significant increase in Cd content started at the lowest amendment rate $10 \mathrm{~g} / \mathrm{kg}$; for $\mathrm{Co}, \mathrm{Mn}$, and $\mathrm{Pb}$, the significant increase was detected at the highest amendment rate $(40 \mathrm{~g} / \mathrm{kg})$. The measured heavy metals in the soil mixtures in all treatments after harvesting pea plants were within the allowed limits reviewed in several studies [34,41,42]. Similar effects of SS amendments on cultivated soil chemical properties were observed in our previous studies on wheat, faba bean, and spinach $[10,14,23]$. 
Table 2. Selected chemical properties of soil at different sewage sludge amendment rates after harvesting Pisum sativum that had been grown for 57 days (means \pm standard error, $n=6$ ).

\begin{tabular}{|c|c|c|c|c|c|c|c|}
\hline \multirow{2}{*}{ Properties } & \multicolumn{5}{|c|}{ Sewage Sludge Amendment Rate (g/kg) } & \multirow[t]{2}{*}{$F$-value } & \multirow{2}{*}{$\begin{array}{c}\text { Maximum } \\
\text { Permissible Limits in } \\
\text { Agricultural Soil }{ }^{+}\end{array}$} \\
\hline & 0 & 10 & 20 & 30 & 40 & & \\
\hline Salinity $(\mathrm{mS} / \mathrm{cm})$ & $0.35 \pm 0.05^{\mathrm{a}}$ & $0.47 \pm 0.03^{a b}$ & $0.52 \pm 0.02 \mathrm{ab}$ & $0.55 \pm 0.05^{b}$ & $0.58 \pm 0.07^{b}$ & $4.0 *$ & NA \\
\hline $\mathrm{pH}$ & $8.38 \pm 0.07^{\mathrm{e}}$ & $7.88 \pm 0.03^{d}$ & $7.63 \pm 0.03^{c}$ & $7.37 \pm 0.03^{b}$ & $7.09 \pm 0.01^{\mathrm{a}}$ & $157.2^{* * *}$ & NA \\
\hline Organic matter $(\%)$ & $1.20 \pm 0.15^{\mathrm{a}}$ & $3.04 \pm 0.11^{\mathrm{b}}$ & $4.66 \pm 0.18^{c}$ & $6.85 \pm 0.26^{\mathrm{d}}$ & $6.78 \pm 0.06^{\mathrm{d}}$ & $215.9^{* * *}$ & NA \\
\hline $\mathrm{Cd}(\mathrm{mg} / \mathrm{kg})$ & $2.21 \pm 0.17^{\mathrm{a}}$ & $3.29 \pm 0.23^{b}$ & $3.39 \pm 0.09^{b}$ & $3.64 \pm 0.03^{b}$ & $3.65 \pm 0.09^{b}$ & $17.9^{* * *}$ & $1.0-5.0$ \\
\hline $\mathrm{Co}(\mathrm{mg} / \mathrm{kg})$ & $26.21 \pm 0.27^{\mathrm{a}}$ & $26.56 \pm 0.10^{a}$ & $28.60 \pm 1.88^{\mathrm{ab}}$ & $28.70 \pm 0.22 \mathrm{ab}$ & $30.86 \pm 0.31^{b}$ & $4.7^{* *}$ & $20.0-50.0$ \\
\hline $\mathrm{Cr}(\mathrm{mg} / \mathrm{kg})$ & $131.34 \pm 3.72^{\mathrm{a}}$ & $134.33 \pm 4.23$ ab & $139.82 \pm 1.40^{\mathrm{ab}}$ & $145.89 \pm 1.13^{b}$ & $146.72 \pm 4.41^{b}$ & $4.3^{* *}$ & $50.0-200.0$ \\
\hline $\mathrm{Cu}(\mathrm{mg} / \mathrm{kg})$ & $16.51 \pm 0.64^{\mathrm{a}}$ & $19.97 \pm 1.30^{\mathrm{a}}$ & $29.37 \pm 0.78^{b}$ & $31.34 \pm 3.03^{b}$ & $31.70 \pm 0.09^{b}$ & $20.9^{* * *}$ & $60.0-150.0$ \\
\hline $\mathrm{Fe}(\mathrm{mg} / \mathrm{g})$ & $38.16 \pm 0.39^{a}$ & $39.88 \pm 0.34^{a}$ & $40.44 \pm 0.82^{\mathrm{a}}$ & $42.74 \pm 1.68^{\mathrm{a}}$ & $42.76 \pm 6.05^{\mathrm{a}}$ & $0.5^{\mathrm{ns}}$ & $20.0-40.0^{++}$ \\
\hline $\mathrm{Mn}(\mathrm{mg} / \mathrm{kg})$ & $587.5 \pm 2.7^{\mathrm{a}}$ & $607.6 \pm 22.3^{\mathrm{ab}}$ & $616.9 \pm 7.2^{a b}$ & $621.6 \pm 6.3^{\mathrm{ab}}$ & $641.2 \pm 2.0^{b}$ & $3.2 *$ & $<450.0 \ddagger$ \\
\hline Mo (mg/kg) & $1.05 \pm 0.12^{\mathrm{a}}$ & $1.07 \pm 0.10^{\mathrm{a}}$ & $1.10 \pm 0.01^{\mathrm{a}}$ & $1.18 \pm 0.02^{\mathrm{a}}$ & $1.19 \pm 0.06^{\mathrm{a}}$ & $1.1^{\mathrm{ns}}$ & $4.0-10.0$ \\
\hline $\mathrm{Ni}(\mathrm{mg} / \mathrm{kg})$ & $31.30 \pm 0.24^{\mathrm{a}}$ & $32.11 \pm 1.25^{\mathrm{a}}$ & $32.71 \pm 0.12^{\mathrm{a}}$ & $34.88 \pm 0.04^{\mathrm{b}}$ & $35.02 \pm 0.36^{\mathrm{b}}$ & $7.8^{* * *}$ & $20.0-60.0$ \\
\hline $\mathrm{Pb}(\mathrm{mg} / \mathrm{kg})$ & $3.27 \pm 0.15^{\mathrm{a}}$ & $3.43 \pm 0.04^{\mathrm{a}}$ & $3.85 \pm 0.11^{\mathrm{a}}$ & $3.97 \pm 0.25^{\mathrm{a}}$ & $4.73 \pm 0.24^{b}$ & $10.5^{* * *}$ & $20.0-30.0$ \\
\hline $\mathrm{Zn}(\mathrm{mg} / \mathrm{kg})$ & $71.18 \pm 0.72^{a}$ & $72.42 \pm 3.70^{a}$ & $100.70 \pm 8.67^{b}$ & $102.08 \pm 0.03^{b}$ & $108.52 \pm 2.02^{b}$ & $16.9^{* * *}$ & $100.0-300.0$ \\
\hline
\end{tabular}

$F$-values represent one-way ANOVA, degrees of freedom $(d f)=4$. Means in the same row followed by different letters are significantly different at $p<0.05$ according to Tukey's HSD test.

${ }^{*}: p<0.05,{ }^{* *}: p<0.01,{ }^{* * *}: p<0.001$, ns: not significant (i.e., $\left.p>0.05\right)$, NA: not applicable, ${ }^{\dagger}$ reviewed by Kabata-Pendias (2011), ${ }^{+\dagger}$ Cornell and Schwertmann (2003), ${ }^{\ddagger}$ Adriano (2001). 
The concentrations of heavy metals in roots, shoots, and fruits of $P$. sativum grown for 57 days in the soils mixed with SS are shown in Table 3. Generally, all heavy metals increased significantly in portions of $P$. sativum except $\mathrm{Cd}$ in the shoot. At an amendment rate of $10 \mathrm{~g} / \mathrm{kg}$, Co in the shoot and root, $\mathrm{Cr}$ in the fruit, $\mathrm{Cu}$ in the root, $\mathrm{Fe}$ in the fruit, $\mathrm{Mn}$ in the shoot and fruit, $\mathrm{Mo}$ in the fruit, $\mathrm{Pb}$ in the shoot, and $\mathrm{Zn}$ in the fruit were elevated significantly. In contrast, the concentrations of $\mathrm{Cd}$ in the fruit, $\mathrm{Cr}$ in the root, $\mathrm{Cu}$ in the shoot, $\mathrm{Fe}$ in the shoot and root, $\mathrm{Ni}$ in the fruit and root, $\mathrm{Pb}$ in the fruit and root, and $\mathrm{Zn}$ in the root significantly increased only at the highest amendment rate. Heavy metals were reported to be accumulated in all parts of plants such as peanut [43] and pea [44]. The results indicate that the heavy metal accumulation in pea portions was below the safe limit set by Codex Alimentarious Commission [45], except for $\mathrm{Cr}, \mathrm{Fe}, \mathrm{Mo}, \mathrm{Ni}, \mathrm{Pb}$ and $\mathrm{Zn}$ in roots. The linear regression estimations of heavy metal content in the different portions of P. sativum over all SS amendment rates indicated that the highest $R^{2}$ values were 0.927 and 0.904 for $\mathrm{Mn}$ and $\mathrm{Cr}$ in fruits, respectively. The lowest $R^{2}$ values were 0.157 and 0.154 for $\mathrm{Mn}$ in roots and Cd in shoots, respectively (Table 4). Translocation of heavy metals by $P$. sativum has been detected in the case of using electroplating industrial sludge that was acidic (like the municipal SS used in this study). The addition of $0.5 \%$ lime as a treatment to the SS minimized the uptake and translocation of the toxic metals in P. sativum plants [46]. 
Table 3. Effects of different amendment rates of sewage sludge on heavy metal concentrations (mg/ $\mathrm{kg}$ ) in fruits, shoots, and roots of Pisum sativum plants that were harvested after 57 days (means \pm standard error, $n=6$ ).

\begin{tabular}{|c|c|c|c|c|c|c|c|c|c|}
\hline \multirow{2}{*}{ Metal } & \multirow{2}{*}{ Tissue } & \multicolumn{5}{|c|}{ Sewage Sludge Amendment Rate (g/kg) } & \multirow{2}{*}{$F$-Value } & \multirow{2}{*}{ Safe Limit + } & \multirow{2}{*}{$\begin{array}{c}\text { Phytotoxic } \\
\text { Range } \ddagger\end{array}$} \\
\hline & & 0 & 10 & 20 & 30 & 40 & & & \\
\hline \multirow{4}{*}{$\mathrm{Cd}$} & Fruit & $0.21 \pm 0.03^{\mathrm{a}}$ & $0.24 \pm 0.03^{a}$ & $0.24 \pm 0.00^{\mathrm{a}}$ & $0.25 \pm 0.00^{\mathrm{a}}$ & $0.51 \pm 0.00^{b}$ & $44.4^{* * *}$ & & \\
\hline & Shoot & $0.24 \pm 0.00^{\mathrm{a}}$ & $0.26 \pm 0.02^{\mathrm{a}}$ & $0.26 \pm 0.02^{\mathrm{a}}$ & $0.27 \pm 0.02^{\mathrm{a}}$ & $0.30 \pm 0.02^{\mathrm{a}}$ & $1.5^{\mathrm{ns}}$ & 0.3 & $5-30$ \\
\hline & Root & $0.42 \pm 0.03^{\mathrm{a}}$ & $0.53 \pm 0.08^{a}$ & $0.68 \pm 0.09 \mathrm{ab}$ & $0.80 \pm 0.01^{b}$ & $0.81 \pm 0.09^{b}$ & $6.0 * *$ & & \\
\hline & Fruit & $1.60 \pm 0.01^{\mathrm{a}}$ & $1.79 \pm 0.13^{\mathrm{ab}}$ & $1.93 \pm 0.03^{b}$ & $3.17 \pm 0.06^{c}$ & $4.60 \pm 0.01 \mathrm{~d}$ & $399.6^{* * *}$ & & \\
\hline \multirow{3}{*}{ Co } & Shoot & $0.84 \pm 0.10^{\mathrm{a}}$ & $1.44 \pm 0.07^{b}$ & $1.51 \pm 0.03^{\mathrm{b}}$ & $1.74 \pm 0.12^{b c}$ & $2.03 \pm 0.09^{c}$ & $25.6^{* * *}$ & - & $30-40$ \\
\hline & Root & $9.66 \pm 0.24^{\mathrm{a}}$ & $13.16 \pm 1.69^{b}$ & $13.96 \pm 0.59^{b}$ & $14.54 \pm 0.12^{b}$ & $14.76 \pm 0.30^{b}$ & $6.5^{* *}$ & & \\
\hline & Fruit & $1.44 \pm 0.02^{\mathrm{a}}$ & $2.52 \pm 0.21^{b}$ & $4.09 \pm 0.25^{c}$ & $4.84 \pm 0.16^{\mathrm{d}}$ & $5.33 \pm 0.07^{\mathrm{d}}$ & $94.9^{* * *}$ & & \\
\hline \multirow[t]{3}{*}{$\mathrm{Cr}$} & Shoot & $2.43 \pm 0.06^{\mathrm{a}}$ & $2.85 \pm 0.13^{a b}$ & $3.63 \pm 0.03 \mathrm{abc}$ & $4.09 \pm 0.74 \mathrm{bc}$ & $5.14 \pm 0.49^{c}$ & $7.0 * *$ & 5 & $10-100$ \\
\hline & Root & $30.64 \pm 4.64^{\mathrm{a}}$ & $43.15 \pm 7.29^{a}$ & $44.29 \pm 3.38^{\mathrm{a}}$ & $45.41 \pm 3.58^{a}$ & $63.39 \pm 2.32^{b}$ & $6.6^{* *}$ & & \\
\hline & Fruit & $4.97 \pm 0.02^{\mathrm{a}}$ & $6.08 \pm 0.04^{\mathrm{a}}$ & $6.94 \pm 0.03^{\mathrm{a}}$ & $9.35 \pm 0.83^{b}$ & $11.83 \pm 0.88^{c}$ & $26.1^{* * *}$ & & \\
\hline \multirow[t]{3}{*}{$\mathrm{Cu}$} & Shoot & $3.48 \pm 0.48^{\mathrm{a}}$ & $4.49 \pm 0.27^{\mathrm{a}}$ & $4.61 \pm 0.04^{\mathrm{a}}$ & $4.70 \pm 0.01^{\mathrm{a}}$ & $8.49 \pm 0.52^{b}$ & $32.7 * * *$ & 40 & 20-100 \\
\hline & Root & $4.48 \pm 0.22^{\mathrm{a}}$ & $14.66 \pm 0.22^{b}$ & $16.00 \pm 1.96^{b c}$ & $17.03 \pm 1.03^{b c}$ & $18.77 \pm 0.19^{c}$ & $31.4^{* * *}$ & & \\
\hline & Fruit & $112.3 \pm 4.2^{\mathrm{a}}$ & $227.7 \pm 39.9^{b}$ & $264.1 \pm 45.3^{b c}$ & $281.9 \pm 5.0^{b c}$ & $347.7 \pm 11.9^{c}$ & $9.8^{* * *}$ & & \\
\hline \multirow[t]{3}{*}{$\mathrm{Fe}$} & Shoot & $266.9 \pm 28.7^{a}$ & $278.5 \pm 43.9^{a}$ & $364.7 \pm 33.2^{\mathrm{a}}$ & $370.4 \pm 76.9^{a}$ & $647.7 \pm 53.7^{b}$ & $9.4^{* * *}$ & 450 & $>1000$ \\
\hline & Root & $8405.6 \pm 1152.4^{a}$ & $12,189.6 \pm 2123.5^{\mathrm{a}}$ & $12,212.5 \pm 890.9^{a}$ & $12,290.8 \pm 910.3^{a}$ & $17,479.6 \pm 251.6^{b}$ & $6.9^{* *}$ & & \\
\hline & Fruit & $43.2 \pm 0.9^{\mathrm{a}}$ & $85.3 \pm 6.9^{b}$ & $93.5 \pm 0.9^{\mathrm{b}}$ & $167.0 \pm 5.0^{c}$ & $218.2 \pm 3.8^{d}$ & $274.8^{* * *}$ & & \\
\hline \multirow[t]{3}{*}{$\mathrm{Mn}$} & Shoot & $95.2 \pm 10.5^{\mathrm{a}}$ & $152.9 \pm 3.5^{b}$ & $158.7 \pm 2.5^{b}$ & $164.4 \pm 0.6^{b}$ & $271.1 \pm 1.2^{c}$ & $157.0^{* * *}$ & - & $>400$ \\
\hline & Root & $366.7 \pm 25.7^{a}$ & $380.1 \pm 2.1^{\mathrm{a}}$ & $391.3 \pm 15.0^{\mathrm{a}}$ & $416.1 \pm 0.5^{\mathrm{a}}$ & $454.1 \pm 61.9^{a}$ & $1.3^{\mathrm{ns}}$ & & \\
\hline & Fruit & $3.23 \pm 0.12^{a}$ & $4.02 \pm 0.01^{b}$ & $4.21 \pm 0.07^{b}$ & $9.83 \pm 0.13^{c}$ & $21.16 \pm 0.21^{\mathrm{d}}$ & $3632.6^{* * *}$ & & \\
\hline \multirow[t]{3}{*}{ Mo } & Shoot & $2.31 \pm 0.11^{\mathrm{a}}$ & $2.34 \pm 0.12^{\mathrm{a}}$ & $4.88 \pm 0.59^{b}$ & $5.20 \pm 0.65^{b}$ & $15.43 \pm 0.84^{c}$ & $97.9^{* * *}$ & 10 & 135 \\
\hline & Root & $1.87 \pm 0.38^{a}$ & $3.16 \pm 0.45^{a b}$ & $3.99 \pm 0.89 a b$ & $4.88 \pm 0.45 b c$ & $6.85 \pm 1.14^{c}$ & $6.7^{* *}$ & & \\
\hline & Fruit & $5.17 \pm 0.04^{\mathrm{a}}$ & $5.20 \pm 0.09^{a}$ & $5.88 \pm 0.60 \mathrm{ab}$ & $6.20 \pm 0.07 \mathrm{ab}$ & $6.57 \pm 0.07^{b}$ & $4.9^{* *}$ & & \\
\hline \multirow[t]{3}{*}{$\mathrm{Ni}$} & Shoot & $2.07 \pm 0.31^{\mathrm{a}}$ & $2.11 \pm 0.08^{a}$ & $2.21 \pm 0.01^{\mathrm{a}}$ & $3.09 \pm 0.13^{b}$ & $3.68 \pm 0.36^{b}$ & $10.3^{* * *}$ & 20 & $40-246$ \\
\hline & Root & $21.07 \pm 1.42^{\mathrm{a}}$ & $22.72 \pm 1.01^{\mathrm{a}}$ & $23.36 \pm 3.11^{a b}$ & $24.49 \pm 0.59 \mathrm{ab}$ & $29.30 \pm 0.08^{b}$ & $3.7 *$ & & \\
\hline & Fruit & $0.39 \pm 0.02^{\mathrm{a}}$ & $0.41 \pm 0.02^{a}$ & $0.44 \pm 0.03^{a}$ & $0.49 \pm 0.05^{\mathrm{a}}$ & $1.02 \pm 0.01^{b}$ & $77.4^{* * *}$ & 5 & $30-300$ \\
\hline \multirow[t]{3}{*}{$\mathrm{Pb}$} & Shoot & $0.38 \pm 0.05^{\mathrm{a}}$ & $3.44 \pm 0.21^{b}$ & $4.09 \pm 0.31^{b}$ & $5.90 \pm 0.44^{c}$ & $6.03 \pm 0.32^{c}$ & $59.6^{* * *}$ & & \\
\hline & Root & $5.05 \pm 0.95^{\mathrm{a}}$ & $6.36 \pm 0.47^{\mathrm{a}}$ & $6.55 \pm 0.56^{a b}$ & $7.83 \pm 1.02^{a b}$ & $9.31 \pm 0.43^{b}$ & $4.9^{* *}$ & & \\
\hline & Fruit & $29.8 \pm 0.3^{\mathrm{a}}$ & $32.0 \pm 0.5^{\mathrm{b}}$ & $33.2 \pm 0.3^{c}$ & $37.2 \pm 0.2^{d}$ & $63.7 \pm 0.2^{\mathrm{e}}$ & $1925.9^{* * *}$ & & \\
\hline \multirow[t]{2}{*}{$\mathrm{Zn}$} & Shoot & $13.6 \pm 1.4^{\mathrm{a}}$ & $18.6 \pm 1.0^{\mathrm{ab}}$ & $19.3 \pm 1.0^{\mathrm{ab}}$ & $24.8 \pm 0.1^{b}$ & $39.2 \pm 3.5^{c}$ & $29.6^{* * *}$ & 60 & $100-500$ \\
\hline & Root & $51.3 \pm 6.1^{\mathrm{a}}$ & $62.9 \pm 1.0^{\mathrm{a}}$ & $69.7 \pm 5.4^{\mathrm{a}}$ & $69.8 \pm 1.8^{\mathrm{a}}$ & $148.2 \pm 20.3^{b}$ & $15.5^{* * *}$ & & \\
\hline
\end{tabular}

$F$-values represent one-way ANOVA, degrees of freedom $(d f)=4$. Means in the same row followed by different letters are significantly different at $p<0.05$, according to Tukey's HSD test.

*: $p<0.05,{ }^{* *}: p<0.01,{ }^{* * *}: p<0.001, n s:$ not significant (i.e., $p>0.05$ ), +: FAO/WHO standard [45] (Codex Alimentarious Commission 2011), †: [34] Kabata-Pendias (2011). 
Table 4. Linear regression equations of the form $y=a+b x$, where $y$ represents the heavy metal concentration $(\mathrm{mg} / \mathrm{kg})$ in Pisum sativum tissue harvested after 57 days, and $x$ is the sewage sludge amendment rate $(\mathrm{g} / \mathrm{kg})$.

\begin{tabular}{|c|c|c|c|c|c|c|c|}
\hline & & $A$ & $S E$ & $B$ & $S E$ & $R^{2}$ & $p$ \\
\hline & Fruit & 0.168 & 0.027 & 0.006 & 0.001 & 0.530 & 0.000 \\
\hline \multirow{3}{*}{$\mathrm{Cd}$} & Shoot & 0.241 & 0.014 & 0.001 & 0.001 & 0.154 & 0.032 \\
\hline & Root & 0.437 & 0.052 & 0.010 & 0.002 & 0.461 & 0.000 \\
\hline & Fruit & 1.142 & 0.152 & 0.074 & 0.006 & 0.833 & 0.000 \\
\hline \multirow[t]{3}{*}{ Co } & Shoot & 0.978 & 0.073 & 0.027 & 0.003 & 0.743 & 0.000 \\
\hline & Root & 10.899 & 0.666 & 0.116 & 0.027 & 0.393 & 0.000 \\
\hline & Fruit & 1.624 & 0.153 & 0.101 & 0.006 & 0.904 & 0.000 \\
\hline \multirow[t]{3}{*}{$\mathrm{Cr}$} & Shoot & 2.299 & 0.297 & 0.066 & 0.012 & 0.518 & 0.000 \\
\hline & Root & 31.819 & 3.617 & 0.678 & 0.148 & 0.429 & 0.000 \\
\hline & Fruit & 4.434 & 0.433 & 0.170 & 0.018 & 0.767 & 0.000 \\
\hline \multirow[t]{3}{*}{$\mathrm{Cu}$} & Shoot & 3.103 & 0.395 & 0.102 & 0.016 & 0.591 & 0.000 \\
\hline & Root & 7.998 & 1.098 & 0.310 & 0.045 & 0.630 & 0.000 \\
\hline & Fruit & 141.751 & 21.614 & 5.250 & 0.882 & 0.558 & 0.000 \\
\hline \multirow[t]{3}{*}{$\mathrm{Fe}$} & Shoot & 214.941 & 42.742 & 8.536 & 1.745 & 0.461 & 0.000 \\
\hline & Root & 8865.751 & 993.829 & 182.494 & 40.573 & 0.419 & 0.000 \\
\hline & Fruit & 35.101 & 5.589 & 4.316 & 0.228 & 0.927 & 0.000 \\
\hline \multirow{3}{*}{$\mathrm{Mn}$} & Shoot & 95.846 & 8.908 & 3.632 & 0.364 & 0.781 & 0.000 \\
\hline & Root & 359.479 & 22.653 & 2.109 & 0.925 & 0.157 & 0.030 \\
\hline & Fruit & 0.155 & 1.086 & 0.417 & 0.044 & 0.759 & 0.000 \\
\hline \multirow[t]{3}{*}{ Mo } & Shoot & 0.212 & 0.934 & 0.291 & 0.038 & 0.676 & 0.000 \\
\hline & Root & 1.815 & 0.539 & 0.117 & 0.022 & 0.502 & 0.000 \\
\hline & Fruit & 5.046 & 0.207 & 0.038 & 0.008 & 0.419 & 0.000 \\
\hline \multirow[t]{3}{*}{$\mathrm{Ni}$} & Shoot & 1.791 & 0.183 & 0.042 & 0.007 & 0.530 & 0.000 \\
\hline & Root & 20.540 & 1.234 & 0.182 & 0.050 & 0.319 & 0.001 \\
\hline & Fruit & 0.278 & 0.052 & 0.014 & 0.002 & 0.595 & 0.000 \\
\hline \multirow[t]{3}{*}{$\mathrm{Pb}$} & Shoot & 1.218 & 0.308 & 0.138 & 0.013 & 0.810 & 0.000 \\
\hline & Root & 5.020 & 0.540 & 0.100 & 0.022 & 0.421 & 0.000 \\
\hline & Fruit & 24.582 & 2.313 & 0.731 & 0.094 & 0.681 & 0.000 \\
\hline \multirow[t]{2}{*}{$\mathrm{Zn}$} & Shoot & 11.580 & 1.722 & 0.575 & 0.070 & 0.705 & 0.000 \\
\hline & Root & 40.256 & 9.673 & 2.006 & 0.395 & 0.480 & 0.000 \\
\hline
\end{tabular}

SE: standard error, $n=30$.

\subsection{Bioaccumulation and Translocation of Heavy Metals in Pea}

BF and TF of heavy metals in harvested P. sativum were calculated (Table 5). TF were assessed for both shoot and fruits, since the plant shoot system could be used as feeding materials for livestock and the fruits are used for human food. The accumulation of $\mathrm{Cd}$ and $\mathrm{Cr}$ was significant only at high SS amendment rates (30 and $40 \mathrm{~g} / \mathrm{kg}$ ). $\mathrm{Co}, \mathrm{Ni}$, and $\mathrm{Pb}$ did not accumulate significantly at any treatment level. The beneficial micronutrient metals such as Fe and $\mathrm{Zn}$ accumulated at a treatment of $40 \mathrm{~g} / \mathrm{kg}$. Mn accumulation was not significant. The TF of heavy metals in the shoot system increased at high amendment rates for $\mathrm{Co}, \mathrm{Cr}, \mathrm{Mn}, \mathrm{Mo}, \mathrm{Ni}$, and $\mathrm{Zn}$. For the fruits, the $\mathrm{TF}$ of $\mathrm{Ni}, \mathrm{Pb}$, and $\mathrm{Zn}$ were not significant at any treatment level, however, $\mathrm{Co}, \mathrm{Cr}, \mathrm{Fe}, \mathrm{Mn}$, and Mo translocate into the fruits at the $30 \mathrm{~g} / \mathrm{kg}$ amendment rate. Bioaccumulation and translocation factors were $>1$ for Mo and the bioaccumulation of $\mathrm{Pb}$ was $>1$ (Table 5). Accumulation of heavy metals in plant shoots at high SS amendment rates interferes with essential physiological processes such as photosynthesis and subsequently affects the growth and yield of the pea plant [36]. Accumulation of heavy metals in the aboveground portions of plants may affect the carbon dioxide fixation and nitrogen metabolism which, ultimately, decrease the growth and development of the plant [25]. Prediction models of heavy metal concentrations in P. sativum grown in soils amended with sewage sludge were published recently by our research group [47]. 
Table 5. Bioaccumulation factors (BFs), from soil to roots, and translocation factors (TFs), from roots to fruits and shoots, of heavy metals in Pisum sativum grown in soil with different sewage sludge amendment rates (means \pm standard error, $n=6$ ).

\begin{tabular}{|c|c|c|c|c|c|c|c|}
\hline \multirow{2}{*}{ Metal } & \multirow{2}{*}{ Factor } & \multicolumn{5}{|c|}{ Sewage Sludge Amendment rate $(\mathrm{g} / \mathrm{kg})$} & \multirow{2}{*}{$F$-Value } \\
\hline & & 0 & 10 & 20 & 30 & 40 & \\
\hline \multirow{4}{*}{$\mathrm{Cd}$} & $\mathrm{BF}$ & $0.194 \pm 0.008^{\mathrm{ab}}$ & $0.156 \pm 0.012^{\mathrm{a}}$ & $0.196 \pm 0.024$ ac & $0.219 \pm 0.005^{b c}$ & $0.219 \pm 0.019 \mathrm{bc}$ & $2.9^{*}$ \\
\hline & $\mathrm{TF}_{\text {shoot }}$ & $0.582 \pm 0.036^{\mathrm{b}}$ & $0.566 \pm 0.108^{b}$ & $0.426 \pm 0.053^{\mathrm{ab}}$ & $0.333 \pm 0.028^{a}$ & $0.384 \pm 0.013^{\mathrm{ab}}$ & $3.7^{*}$ \\
\hline & $\mathrm{TF}_{\text {fruit }}$ & $0.501 \pm 0.071 \mathrm{ab}$ & $0.497 \pm 0.083^{\mathrm{ab}}$ & $0.404 \pm 0.059^{\mathrm{a}}$ & $0.308 \pm 0.003^{\mathrm{a}}$ & $0.675 \pm 0.077^{\mathrm{b}}$ & $4.3^{* *}$ \\
\hline & $\mathrm{BF}$ & $0.368 \pm 0.005^{\mathrm{a}}$ & $0.495 \pm 0.063^{\mathrm{a}}$ & $0.506 \pm 0.054^{\mathrm{a}}$ & $0.507 \pm 0.001^{\mathrm{a}}$ & $0.478 \pm 0.007^{\mathrm{a}}$ & $2.5^{\mathrm{ns}}$ \\
\hline \multirow[t]{3}{*}{ Co } & $\mathrm{TF}_{\text {shoot }}$ & $0.086 \pm 0.009^{\mathrm{a}}$ & $0.116 \pm 0.010^{a b}$ & $0.108 \pm 0.003^{\mathrm{ab}}$ & $0.120 \pm 0.009^{b}$ & $0.138 \pm 0.009^{b}$ & $5.3 * *$ \\
\hline & $\mathrm{TF}_{\text {fruit }}$ & $0.166 \pm 0.003^{b}$ & $0.141 \pm 0.009^{\mathrm{a}}$ & $0.139 \pm 0.008^{\mathrm{a}}$ & $0.218 \pm 0.006^{c}$ & $0.312 \pm 0.007^{\mathrm{d}}$ & $113.6^{* * *}$ \\
\hline & $\mathrm{BF}$ & $0.239 \pm 0.042^{\mathrm{a}}$ & $0.331 \pm 0.065^{\mathrm{ab}}$ & $0.316 \pm 0.021^{\mathrm{ab}}$ & $0.312 \pm 0.027^{\mathrm{ab}}$ & $0.432 \pm 0.009^{b}$ & $3.3 *$ \\
\hline \multirow[t]{3}{*}{$\mathrm{Cr}$} & $\mathrm{TF}_{\text {shoot }}$ & $0.091 \pm 0.016^{\mathrm{a}}$ & $0.074 \pm 0.009^{a}$ & $0.084 \pm 0.007^{\mathrm{a}}$ & $0.086 \pm 0.009^{\mathrm{a}}$ & $0.080 \pm 0.005^{\mathrm{a}}$ & $0.4^{\mathrm{ns}}$ \\
\hline & $\mathrm{TF}_{\text {fruit }}$ & $0.053 \pm 0.008^{a}$ & $0.063 \pm 0.006^{\mathrm{ab}}$ & $0.093 \pm 0.001^{c}$ & $0.112 \pm 0.012^{c}$ & $0.085 \pm 0.004 b c$ & $10.3^{* * *}$ \\
\hline & $\mathrm{BF}$ & $0.276 \pm 0.024^{\mathrm{a}}$ & $0.746 \pm 0.038^{b}$ & $0.556 \pm 0.082^{b}$ & $0.587 \pm 0.089^{b}$ & $0.592 \pm 0.006^{\mathrm{b}}$ & $8.8^{* * *}$ \\
\hline \multirow[t]{3}{*}{$\mathrm{Cu}$} & $\mathrm{TF}_{\text {shoot }}$ & $0.759 \pm 0.071^{c}$ & $0.308 \pm 0.023^{\mathrm{ab}}$ & $0.309 \pm 0.035^{\mathrm{ab}}$ & $0.281 \pm 0.017^{\mathrm{a}}$ & $0.451 \pm 0.023^{b}$ & $26.4^{* * *}$ \\
\hline & $\mathrm{TF}_{\text {fruit }}$ & $1.122 \pm 0.055^{c}$ & $0.415 \pm 0.008^{a}$ & $0.468 \pm 0.056^{\mathrm{a}}$ & $0.545 \pm 0.016^{\mathrm{ab}}$ & $0.628 \pm 0.040^{\mathrm{b}}$ & $49.9^{* * *}$ \\
\hline & $\mathrm{BF}$ & $0.222 \pm 0.032^{\mathrm{a}}$ & $0.304 \pm 0.051^{\mathrm{ab}}$ & $0.305 \pm 0.028^{a b}$ & $0.294 \pm 0.033^{a b}$ & $0.452 \pm 0.069^{b}$ & $3.5 *$ \\
\hline \multirow[t]{3}{*}{$\mathrm{Fe}$} & $\mathrm{TF}_{\text {shoot }}$ & $0.038 \pm 0.009^{\mathrm{a}}$ & $0.023 \pm 0.001^{a}$ & $0.030 \pm 0.001^{\mathrm{a}}$ & $0.029 \pm 0.004^{\mathrm{a}}$ & $0.037 \pm 0.003^{\mathrm{a}}$ & $1.9^{\mathrm{ns}}$ \\
\hline & $\mathrm{TF}_{\text {fruit }}$ & $0.014 \pm 0.002^{\mathrm{a}}$ & $0.019 \pm 0.000^{\mathrm{ab}}$ & $0.021 \pm 0.002^{b}$ & $0.024 \pm 0.002^{b}$ & $0.020 \pm 0.001 \mathrm{ab}$ & $4.7^{* *}$ \\
\hline & $\mathrm{BF}$ & $0.625 \pm 0.046^{\mathrm{a}}$ & $0.629 \pm 0.019^{a}$ & $0.639 \pm 0.032^{a}$ & $0.669 \pm 0.007^{\mathrm{a}}$ & $0.709 \pm 0.099^{a}$ & $0.5^{\mathrm{ns}}$ \\
\hline \multirow[t]{3}{*}{ Mn } & $\mathrm{TF}_{\text {shoot }}$ & $0.256 \pm 0.011^{\mathrm{a}}$ & $0.403 \pm 0.011^{\mathrm{a}}$ & $0.407 \pm 0.009^{\mathrm{a}}$ & $0.395 \pm 0.002^{\mathrm{a}}$ & $0.656 \pm 0.087^{b}$ & $13.3^{* * *}$ \\
\hline & $\mathrm{TF}_{\text {fruit }}$ & $0.122 \pm 0.011^{\mathrm{a}}$ & $0.224 \pm 0.017^{a}$ & $0.241 \pm 0.012^{a}$ & $0.401 \pm 0.012^{b}$ & $0.536 \pm 0.081^{b}$ & $18.2^{* * *}$ \\
\hline & $\mathrm{BF}$ & $1.697 \pm 0.174^{\mathrm{a}}$ & $2.942 \pm 0.420 \mathrm{ab}$ & $3.591 \pm 0.776^{a b c}$ & $4.121 \pm 0.346^{b c}$ & $5.609 \pm 0.687^{c}$ & $7.5^{* * *}$ \\
\hline \multirow[t]{3}{*}{ Mo } & $\mathrm{TF}_{\text {shoot }}$ & $1.642 \pm 0.399 \mathrm{ab}$ & $0.854 \pm 0.159^{a}$ & $1.845 \pm 0.559 \mathrm{ab}$ & $1.176 \pm 0.241^{\mathrm{ab}}$ & $2.496 \pm 0.292^{b}$ & $3.1 *$ \\
\hline & $\mathrm{TF}_{\text {fruit }}$ & $2.256 \pm 0.531 \mathrm{ab}$ & $1.420 \pm 0.205^{\mathrm{a}}$ & $1.425 \pm 0.332^{\mathrm{a}}$ & $2.089 \pm 0.166^{a b}$ & $3.554 \pm 0.560^{\mathrm{b}}$ & $4.9^{* *}$ \\
\hline & $\mathrm{BF}$ & $0.675 \pm 0.051^{\mathrm{a}}$ & $0.719 \pm 0.059^{a}$ & $0.712 \pm 0.093^{\mathrm{a}}$ & $0.702 \pm 0.017^{\mathrm{a}}$ & $0.837 \pm 0.010^{\mathrm{a}}$ & $1.3^{\mathrm{ns}}$ \\
\hline \multirow[t]{3}{*}{$\mathrm{Ni}$} & $\mathrm{TF}_{\text {shoot }}$ & $0.095 \pm 0.008^{\mathrm{a}}$ & $0.093 \pm 0.001^{\mathrm{a}}$ & $0.104 \pm 0.014^{\mathrm{ab}}$ & $0.126 \pm 0.003^{b}$ & $0.125 \pm 0.012^{\mathrm{ab}}$ & $3.2 *$ \\
\hline & $\mathrm{TF}_{\text {fruit }}$ & $0.251 \pm 0.015^{\mathrm{a}}$ & $0.230 \pm 0.006^{a}$ & $0.257 \pm 0.009^{a}$ & $0.254 \pm 0.009^{a}$ & $0.224 \pm 0.003^{a}$ & $2.6^{\mathrm{ns}}$ \\
\hline & $\mathrm{BF}$ & $1.624 \pm 0.360^{\mathrm{a}}$ & $1.862 \pm 0.154^{\mathrm{a}}$ & $1.730 \pm 0.195^{\mathrm{a}}$ & $2.092 \pm 0.382^{\mathrm{a}}$ & $2.014 \pm 0.183^{a}$ & $0.5^{\mathrm{ns}}$ \\
\hline \multirow[t]{3}{*}{$\mathrm{Pb}$} & $\mathrm{TF}_{\text {shoot }}$ & $0.081 \pm 0.007^{\mathrm{a}}$ & $0.557 \pm 0.059^{b}$ & $0.668 \pm 0.109^{b}$ & $0.877 \pm 0.209^{b}$ & $0.658 \pm 0.054^{b}$ & $7.1^{* *}$ \\
\hline & $\mathrm{TF}_{\text {fruit }}$ & $0.095 \pm 0.019^{\mathrm{a}}$ & $0.067 \pm 0.008^{a}$ & $0.071 \pm 0.010^{\mathrm{a}}$ & $0.075 \pm 0.018^{\mathrm{a}}$ & $0.111 \pm 0.005^{\mathrm{a}}$ & $1.9^{\mathrm{ns}}$ \\
\hline & $\mathrm{BF}$ & $0.726 \pm 0.094^{a}$ & $0.884 \pm 0.059^{a}$ & $0.743 \pm 0.118^{a}$ & $0.683 \pm 0.017^{a}$ & $1.386 \pm 0.213^{b}$ & $5.9^{* *}$ \\
\hline \multirow[t]{2}{*}{$\mathrm{Zn}$} & $\mathrm{TF}_{\text {shoot }}$ & $0.267 \pm 0.006^{a}$ & $0.296 \pm 0.020^{\mathrm{ab}}$ & $0.292 \pm 0.037^{\mathrm{ab}}$ & $0.357 \pm 0.008^{b}$ & $0.274 \pm 0.014^{\mathrm{a}}$ & $3.1^{*}$ \\
\hline & $\mathrm{TF}_{\text {fruit }}$ & $0.629 \pm 0.081^{a}$ & $0.508 \pm 0.001^{\mathrm{a}}$ & $0.493 \pm 0.043^{a}$ & $0.535 \pm 0.012^{\mathrm{a}}$ & $0.474 \pm 0.064^{\mathrm{a}}$ & $1.5^{\mathrm{ns}}$ \\
\hline \multicolumn{2}{|c|}{$F$-value $e_{B F}$} & $17.7^{* * *}$ & $33.4^{* * *}$ & $15.6^{* * *}$ & $53.2 * * *$ & $46.0 * * *$ & \\
\hline \multicolumn{2}{|c|}{ F-value TFshoot } & $15.1^{* * *}$ & $17.3^{* * *}$ & $8.7^{* * *}$ & $13.4^{* * *}$ & $54.3^{* * *}$ & \\
\hline \multicolumn{2}{|c|}{ F-value $_{\text {TFfruit }}$} & $16.4^{* * *}$ & $34.5^{* * *}$ & $14.0^{* * *}$ & $126.4^{* * *}$ & $32.7^{* * *}$ & \\
\hline
\end{tabular}

$F$-values represent one-way ANOVA, degrees of freedom $(d f)=4$. Means in the same row followed by different letters are significantly different at $p<0.05$, according to Tukey's HSD test. *: $p<0.05,{ }^{* *}: p<0.01,^{* * *}: p<0.001, n s$ : not significant (i.e., $p>0.05$ ). 
The results indicate that SS could be used to improve agricultural soil quality only with low amendment rates of 10 and $20 \mathrm{~g} / \mathrm{kg}$. Frequent use of SS should be taken in account for long-term environmental and health hazard management.

\section{Materials and Methods}

\subsection{Plant Materials, Sewage Sludge Treatments, and Experimental Design}

Seeds of Pisum sativum (Mashhour Company, Menoufiya Governorate, Egypt) were obtained from a local market in Abha city, Saudi Arabia. The agricultural soil used in the experiment was collected at a depth of $0-20 \mathrm{~cm}$ from neighboring cultivated fields. The SS was obtained from the Abha municipal sewage treatment plant (AMSTP), Aseer region, Saudi Arabia. The AMSTP treats around $41,275 \mathrm{~m}^{3}$ of urban wastewater per day using aerobic tertiary treatment, and the equivalent dry sewage sludge production was assessed as 90 tons per day with daily dumping. The agricultural soil and SS samples were prepared as described previously [16]. The experiment was performed in the greenhouse of the Biology Department, King Khalid University, Abha, Saudi Arabia.

The SS was mixed with agricultural soil at rates of zero (the control soil), 10, 20, 30, and $40 \mathrm{~g} / \mathrm{kg}$. Each treatment consisted of 6 replicates of plastic pots (6 L volume) filled with $4 \mathrm{~kg} /$ pot of the respective treatment. Ten seeds of $P$. sativum were planted in each pot. The experimental units were arranged in a randomized design. The plants were grown for 57 days (starting from 4 January 2018) in the greenhouse with a natural day/night regime and irrigated as needed and weed controlled manually. After 15 days, the plants were counted and then manually thinned to one plant/pot.

\subsection{Plant Morphology and Biomass Measurements}

The shoot heights and root and pod lengths were measured, and the number of leaves and fruits (pods) per individual were counted. The germination percentage was calculated by dividing the germinated seed in each pot by the total seed used in planting. The plant materials were washed under running water, and divided into roots, pods, and shoots (stems and leaves). The partitioned plant materials were dried at $60^{\circ} \mathrm{C}$ for one week and then ground in a plastic mill and stored. The above-ground biomass refers to the aerial portions of the plant, i.e., the sum of the pod and shoot biomass, while the total biomass refers to the sum of the shoot and root biomass. The absolute growth rate (AGR) was obtained as described by Radford [48].

\subsection{Sample Analysis}

For physicochemical analyses, the soil and SS samples were air-dried for two weeks and then ground and sieved through a $2 \mathrm{~mm}$ sieve. The dried samples were analyzed for organic matter content using a loss-on-ignition method at $550{ }^{\circ} \mathrm{C}$ for $2 \mathrm{~h}$. [49]. Electrical conductivity (EC) and $\mathrm{pH}$ were measured in soil-water extracts at 1:5 [50]. For quantifying heavy metals (in 0.5-1.0 g of each sample), soil, SS or plant samples were digested using a mixed-acid digestion method $\left(\mathrm{HNO}_{3}\right.$ and $\mathrm{HClO}_{4} ; 3: 1, \mathrm{v} / \mathrm{v}$ ). A microwave sample preparation system was used for digestion (PerkinElmer Titan MPS, PerkinElmer Inc., Waltham, MA, USA). Blank samples were included to confirm procedure accuracy. Various heavy metals, including $\mathrm{Co}, \mathrm{Cu}, \mathrm{Fe}, \mathrm{Mn}, \mathrm{Ni}, \mathrm{Zn}, \mathrm{Cd}, \mathrm{Cr}$, and $\mathrm{Pb}$, were determined by inductively coupled plasma optical emission spectrometry (ICP-OES) (Thermo Scientific iCAP 7000 Plus Series; Thermo Fisher Scientific, Waltham, MA, USA) according to procedures outlined by [50]. The detection limits of heavy metals (in $\mu \mathrm{g} \mathrm{L}-1$ ) were as follows: 6.0 for $\mathrm{Ni} ; 2.0$ for $\mathrm{Co}, \mathrm{Cr}$, and $\mathrm{Cu}$; 1.0 for $\mathrm{Fe}, \mathrm{Pb}$, and $\mathrm{Zn} ; 0.3$ for $\mathrm{Mn}$; and 0.1 for $\mathrm{Cd}$. The instrument setting and operational conditions followed the manufacturer's specifications. Standard solutions with known concentrations of different heavy metals were prepared for the standardization of the system. A certified reference (SRM 1573a, tomato leaves) was used to verify the accuracy of the heavy metal determination. This reference material was digested and analyzed using the same methods as those applied to the P. sativum samples. Three independent biological replicates were performed for digestion and heavy metal quantification. 
Accuracy was estimated by comparing the measured concentration with the certified value and then expressed as a percentage. The recovery rates were $94.9 \%$ for $\mathrm{Cd}$ with a relative standard deviation $(\mathrm{RSD})=4.3 \%, 104.4 \%$ for $\mathrm{Co}(\mathrm{RSD}=8.0 \%), 95.7 \%$ for $\mathrm{Cr}(\mathrm{RSD}=6.2 \%), 101.6 \%$ for $\mathrm{Cu}(\mathrm{RSD}=3.3 \%)$, $102.9 \%$ for $\mathrm{Fe}(\mathrm{RSD}=4.9 \%), 97.1 \%$ for $\mathrm{Mn}(\mathrm{RSD}=7.4 \%), 98.9 \%$ for $\mathrm{Mo}(\mathrm{RSD}=3.8 \%), 95.2 \%$ for $\mathrm{Ni}$ $(\mathrm{RSD}=9.0 \%), 103.5 \%$ for $\mathrm{Pb}(\mathrm{RSD}=5.6 \%)$ and $97.5 \%$ for $\mathrm{Zn}(\mathrm{RSD}=4.6 \%)$. The bioaccumulation factor (BF) and the translocation factor (TF) were calculated following the method of Ghosh and Singh [51]. $\mathrm{BF}$ is the concentration of a heavy metal (HM) in the roots $(\mathrm{mg} / \mathrm{kg}) /$ concentration of the same HM in soil ( $\mathrm{mg} / \mathrm{kg})$. TF is the concentration of an HM in the above-ground tissue $(\mathrm{mg} / \mathrm{kg}) /$ concentration of the same HM in the roots $(\mathrm{mg} / \mathrm{kg})$.

\subsection{Data Statistical Analyses}

The data were examined for their homogeneity of variance and normality of distribution and, when required, the data were log-transformed before a one-way analysis of variance (ANOVA) was performed. Significant differences in the soil characteristics, biomass, plant morphometric parameters, and heavy metals (HMs) data for the P. sativum tissues, BFs, and TFs across all treatments, and the variation in BFs and TFs within $10 \mathrm{HMs}$ through the same amendment rate of SS, were evaluated using one-way ANOVA. Tukey's honest significant difference (HSD) test at $p<0.05$ was used to identify the significant differences among the means of all treatments. A quadratic regression analysis [52] was performed to evaluate the response of the biomass and plant morphometric parameters of $P$. sativum grown in soils amended with different rates of SS. To assess the statistical relationships among the HM concentrations in P. sativum tissues $(\mathrm{mg} / \mathrm{kg}$ ) and the amendment rate of $\mathrm{SS}(\mathrm{g} / \mathrm{kg})$, regression procedures were applied. Statistica 7.1 was used to perform all statistical analyses [53].

\section{Conclusions}

Sewage sludge (SS) application increases agricultural soil's fertility and plant productivity when used in a suitable rate of soil amendment. The current study focused on the assessment of evaluating heavy metal bioaccumulation, growth, and yield of the legume crop P. sativum grown in agricultural soil mixed with SS at rates of zero, 10, 20, 30, and $40 \mathrm{~g} / \mathrm{kg}$. SS amendments affected seed germination of $P$. sativum severely. The inhibition of seed germination could be due to heavy metal content in SS. Heavy metals were reported as suppressors of mitotic division of plant cells. Growth parameters, i.e., root, shoot, and pod length and biomass, number of leaves and pods, increased at the lower rates of 10 and $20 \mathrm{~g} / \mathrm{kg}$; growth parameters decreased at the higher rates of 30 and $40 \mathrm{~g} / \mathrm{kg}$. The negative effects of a high rate of SS application on plant growth may refer to the toxic effects of heavy metals which include nutritional imbalance caused by the displacement of essential cations with heavy metals, interaction with sulfhydryl groups of functional proteins, decreasing chlorophyll content, affecting hormonal balance and generating reactive oxygen species in plant cells. SS salinity and organic content were greater than that of the cultivated soil used in the experiment. The accumulation trend of heavy metals in plant portions differed according to the amendment rate. Heavy metals in the postharvest soil samples increased for all SS application rates except Fe and Mo. The significant increase in Cd content started at the lowest amendment rate $10 \mathrm{~g} / \mathrm{kg}$; for $\mathrm{Co}, \mathrm{Mn}$, and $\mathrm{Pb}$, the significant increase was detected at the highest amendment rate $(40 \mathrm{~g} / \mathrm{kg})$. Generally, all heavy metals increased significantly in portions of $P$. sativum except $\mathrm{Cd}$ in the shoot. At an amendment rate of $10 \mathrm{~g} / \mathrm{kg}$, Co in the shoot and root, $\mathrm{Cr}$ in the fruit, $\mathrm{Cu}$ in the root, $\mathrm{Fe}$ in the fruit, $\mathrm{Mn}$ in the shoot and fruit, $\mathrm{Mo}$ in the fruit, $\mathrm{Pb}$ in the shoot, and $\mathrm{Zn}$ in the fruit were elevated significantly. In contrast, the concentrations of $\mathrm{Cd}$ in the fruit, $\mathrm{Cr}$ in the root, $\mathrm{Cu}$ in the shoot, $\mathrm{Fe}$ in the shoot and root, $\mathrm{Ni}$ in the fruit and root, $\mathrm{Pb}$ in the fruit and root, and $\mathrm{Zn}$ in the root significantly increased only at the highest rate of $40 \mathrm{~g} / \mathrm{kg}$. The regression $R^{2}$ value was 0.927 for the micronutrient $\mathrm{Mn}$ in the pod and 0.154 for the heavy metal $\mathrm{Cd}$ in the shoot. Low metal translocation was detected for all measured heavy metals. Bioaccumulation and translocation factors were $>1$ for Mo and the bioaccumulation of $\mathrm{Pb}$ was $>1$. The suitable level of SS application into agricultural soil as fertilizer may differ according to the soil $\mathrm{pH}$ and the grown plant species. 
Considering the current study, the application rate of SS as a fertilizer for pea plants should not exceed $20 \mathrm{~g} / \mathrm{kg}$ to avoid environmental and health risks for animals and humans.

Author Contributions: Conceptualization, E.M.E.; methodology, E.M.E., M.A.T., S.A.A. and M.T.A.; software, E.M.E.; formal analysis, E.M.E., M.A.T., S.A.A. and M.T.A.; investigation, E.M.E., M.A.T., S.A.A. and M.T.A.; resources, M.A.T.; data curation, E.M.E.; writing-original draft preparation, A.F.E.-B.; writing-review and editing, T.M.G., K.H.S. and N.A.S.; visualization, E.M.E.; supervision, E.M.E.; project administration, M.A.T.; funding acquisition, M.A.T. All authors have read and agreed to the published version of the manuscript.

Funding: This research was funded by the Deanship of Scientific Research at King Khalid University, grant number R.G.P. 1/73/40 and the APC was funded by the Deanship of Scientific Research at King Khalid University.

Acknowledgments: This work was supported by the Deanship of Scientific Research at King Khalid University under Grant number R.G.P. 1/73/40.

Conflicts of Interest: The authors declare no conflict of interest.

\section{References}

1. Tomócsik, A.; Makádi, M.; Orosz, V.; Aranyos, T.; Demeter, I.; Mészáros, J.; Füleky, G. Effect of sewage sludge compost treatment on crop yield. AGROFOR Int. J. 2016, 1, 5-12. [CrossRef]

2. Otobbang, E.; Sadovnikova, L.; Lakimenko, O.; Nilsson, I.; Persson, J. Sewage sludge: Soil conditioner and nutrient source II. Availability of $\mathrm{Cu}, \mathrm{Zn}, \mathrm{Pb}$, and $\mathrm{Cd}$ to barley in a pot experiment. Acta Agric. Scand. Sect. B Soil Plant Sci. 1997, 47, 65-70. [CrossRef]

3. Gascó, G.; Lobo, M.C. Comparison of a Spanish sewage sludge and effects on treated soil and olive trees. Waste Manag. 2007, 27, 1494-1500. [CrossRef] [PubMed]

4. Antolín, M.C.; Muro, I.; Sánchez-Díaz, M. Application of sewage sludge improves growth, photosynthesis and antioxidant activities of nodulated alfalfa plants under drought conditions. Environ. Exp. Bot. 2010,68, 75-82. [CrossRef]

5. Singh, R.P.; Agrawal, M. Biochemical and physiological responses of rice (Oryza sativa L.) grown on different sewage sludge amendments rates. Bull. Environ. Contam. Toxicol. 2010, 84, 606-612. [CrossRef]

6. Grotto, D.; Batista, B.L.; Souza, J.M.O.; Carneiro, M.F.H.; dos Santos, D.; Melo, W.J.; Barbosa, F., Jr. Essential and nonessential element translocation in corn cultivated under sewage sludge application and associated health risk. Water Air Soil Pollut. 2015, 226, 261. [CrossRef]

7. Koutroubas, S.D.; Antoniadis, V.; Fotiadis, S.; Damalas, C.A. Growth, grain yield and nitrogen use efficiency of Mediterranean wheat in soils amended with municipal sewage sludge. Nutr. Cycl. Agroecosyst. 2014, 100, 227-243. [CrossRef]

8. Tsadilas, C.; Samaras, V.; Evangelou, E.; Shaheen, S.M. Influence of fly ash and sewage sludge application on wheat biomass production, nutrients availability, and soil properties. Int. J. Coal. Sci. Technol. 2014, 1, 221-226. [CrossRef]

9. Yilmaz, D.D.; Temizgül, A. Determination of heavy-metal concentration with chlorophyll contents of wheat (Triticum aestivum) exposed to municipal sewage sludge doses. Commun. Soil Sci. Plant 2014, 45, 2754-2766. [CrossRef]

10. Eid, E.M.; Alrumman, S.A.; El-Bebany, A.F.; Fawy, K.F.; Taher, M.A.; Hesham, A.; El-Shaboury, G.A.; Ahmed, M.T. Evaluation of the potential of sewage sludge as a valuable fertilizer for wheat (Triticum aestivum L.) crops. Environ. Sci. Pollut. Res. 2019, 26, 392-401. [CrossRef]

11. Soriano-Disla, J.M.; Gómez, I.; Navarro-Pedreño, J.; Jordán, M.M. The transfer of heavy metals to barley plants from soils amended with sewage sludge with different heavy metal burdens. J. Soils Sediments 2014, 14, 687-696. [CrossRef]

12. Fernández, J.M.; Plaza, C.; García-Gil, J.C.; Polo, A. Biochemical properties and barley yield in a semiarid Mediterranean soil amended with two kinds of sewage sludge. Appl. Soil Ecol. 2009, 42, 18-24. [CrossRef]

13. Antoniadis, V.; Tsadilas, C.D.; Samaras, V. Trace element availability in a sewage sludge-amended cotton grown Mediterranean soil. Chemosphere 2010, 80, 1308-1313. [CrossRef] [PubMed]

14. Eid, E.M.; Alrumman, S.A.; El-Bebany, A.F.; Fawy, K.F.; Taher, M.A.; Hesham, A.; El-Shaboury, G.A.; Ahmed, M.T. The evaluation of sewage sludge application as a fertilizer for broad bean (Faba sativa Bernh.) crops. Food Energy Secur. 2018, 7, e00142. [CrossRef] 
15. Kumar, V.; Chopra, A.K. Accumulation and translocation of metals in soil and different parts of French bean (Phaseolus vulgaris L.) amended with sewage sludge. Bull. Environ. Contam. Toxicol. 2014, 92, $103-108$. [CrossRef]

16. Eid, E.M.; Alrumman, S.A.; El-Bebany, A.F.; Hesham, A.; Taher, M.A.; Fawy, K.F. The effects of different sewage sludge amendment rates on the heavy metal bioaccumulation, growth and biomass of cucumbers (Cucumis sativus L.). Environ. Sci. Pollut. Res. 2017, 24, 16371-16382. [CrossRef] [PubMed]

17. Waqas, M.; Khan, S.; Qing, H.; Reid, B.J.; Chao, C. The effects of sewage sludge and sewage sludge biochar on PAHs and potentially toxic element bioaccumulation in Cucumis sativa L. Chemosphere 2014, 105, 53-61. [CrossRef]

18. Hossain, M.K.; Strezov, V.; Chan, K.Y.; Nelson, P.F. Agronomic properties of wastewater sludge biochar and bioavailability of metals in production of cherry tomato (Lycopersicon esculentum). Chemosphere 2010, 78, 1167-1171. [CrossRef]

19. Casado-Vela, J.; Sellés, S.; Díaz-Crespo, C.; Navarro-Pedreňo, J.; Mataix-Beneyto, J.; Gómez, I. Effect of composted sewage sludge application to soil on sweet pepper crop (Capsicum annuum var. annuum) grown under two exploitation regimes. Waste Manag. 2007, 27, 1509-1518. [CrossRef]

20. Antonious, G.F.; Kochhar, T.S.; Coolong, T. Yield, quality, and concentration of seven heavy metals in cabbage and broccoli grown in sewage sludge and chicken manure amended soil. J. Environ. Sci. Health A 2012, 47, 1955-1965. [CrossRef]

21. Türkmen, Ö.; Sensoy, S.; Dursun, A.; Turan, M. Sewage sludge as a substitute for mineral fertilization of spinach (Spinacia oleraceae L.) at two growing periods. Acta Agric. Scand. B Soil Plant Sci. 2004, 54, 102-107.

22. Kumar, V.; Chopra, A.K.; Srivastava, S. Assessment of heavy metals in spinach (Spinacia oleracea L.) grown in sewage sludge-amended soil. Commun. Soil Sci. Plant Anal. 2016, 47, 221-236. [CrossRef]

23. Eid, E.M.; El-Bebany, A.F.; Alrumman, S.A.; Hesham, A.; Taher, M.A.; Fawy, K.F. Effects of different sewage sludge applications on heavy metal accumulation, growth and yield of spinach (Spinacia oleracea L.). Int. J. Phytoremed. 2017, 19, 340-347. [CrossRef] [PubMed]

24. Binder, D.L.; Dobermann, A.; Sander, D.H.; Cassman, K.G. Biosolids as nitrogen source for irrigated maize and rainfed sorghum. Soil Sci. Soc. Am. J. 2002, 66, 531-542. [CrossRef]

25. Singh, S.; Parihar, P.; Singh, R.; Singh, V.P.; Prasad, S.M. Heavy metal tolerance in plants: Role of transcriptomics, proteomics, metabolomics, and ionomics. Front. Plant Sci. 2016, 6, 1143. [CrossRef] [PubMed]

26. Husaini, Y.; Rai, L.C. Studies on nitrogen and phosphorus metabolism and the photosynthetic electron transport system of Nostoc linckia under cadmium stress. J. Plant Physiol. 1991, 138, 429-435. [CrossRef]

27. Sharma, S.S.; Dietz, K.J. The relationship between metal toxicity and cellular redox imbalance. Trends Plant Sci. 2009, 14, 43-50. [CrossRef]

28. Singh, J.; Laura, J.S. Effect of sewage irrigation on yield of pea and pigeon pea. J. Integr. Sci. Technol. 2014, 2, $80-84$.

29. Siddiqi, I.; Javaid, A.; Bajwa, R. Sewage farming and VA mycorrhiza III: Effect of sewage irrigation on growth, yield, nodulation and VA mycorrhizal colonization in pea (Pisum sativum L.). Pak. J. Biol. Sci. 2000, 3, 967-968.

30. Sinha, S.N.; Paul, D. Impact of sewage water on seed germination and vigour index of Cicer arietinum L. and Pisum Sativum L. Int. J. Food Agric. Vet. Sci. 2013, 3, 19-26.

31. Lewis, J.A.; Lumsden, R.D.; Millner, P.D.; Keinath, A.P. Suppression of damping-off of peas and cotton in the field with composted sewage sludge. Crop Prot. 1992, 11, 260-266. [CrossRef]

32. Lumsden, R.D.; Lewis, J.A.; Millner, P.D. Effect of sewage sludge on several soilborne pathogens and diseases. Phytopathology 1983, 73, 1543-1548. [CrossRef]

33. He, Z.L.; Yang, X.E.; Stoffella, P.J. Trace elements in agroecosystems and impacts on the environment. J. Trace Elem. Med. Biol. 2005, 19, 125-140. [CrossRef] [PubMed]

34. Kabata-Pendias, A. Trace Elements in Soils and Plants; CRC Press: Boca Raton, FL, USA, 2011.

35. Pandey, S.K. Germination and seedling growth of field pea Pisum sativum Malviya Matar-15(HUDP-15) and Pusa Prabhat (DDR-23) under varying level of copper and chromium. J. Am. Sci. 2008, 4, 28-40.

36. Khan, M.G.; Daniel, G.; Konjit, M.; Thomas, A.; Eyasu, S.S.; Awoke, G. Impact of textile waste water on seed germination and some physiological parameters in pea (Pisum sativum L.), lentil (Lens esculentum L.) and gram (Cicer arietinum L.). Asian J. Plant Sci. 2011, 10, 269-273. [CrossRef] 
37. Burzynski, M. The influence of lead and cadmium on the absorption and distribution of potassium, calcium, magnesium and iron in cucumber seedlings. Acta Physiol. Plant. 1987, 9, 229-238.

38. Larsson, E.H.; Asp, H.; Bornman, J.F. Influence prior $\mathrm{Cd}^{+2}$ exposure on the uptake of $\mathrm{Cd}^{+2}$ and other elements in the phytochelatin-deficient mutant, cad1-3, of Arabidopsis thaliana. J. Exp. Bot. 2002, 53, 447-453. [CrossRef] [PubMed]

39. Khan, A.; Khan, S.; Khan, M.A.; Qamar, Z.; Waqas, M. The uptake and bioaccumulation of heavy metals by food plants, their effects on plants nutrients, and associated health risk: A review. Environ. Sci. Pollut. Res. 2015, 22, 13772-13799. [CrossRef]

40. Chandra, R.; Yadav, S.; Mohan, D. Effect of distillery sludge on seed germination and growth parameters of green gram (Phaseolus mungo L.). J. Hazard Mater. 2008, 152, 431-439. [CrossRef]

41. Cornell, R.M.; Schwertmann, U. The Iron Oxides, 2nd ed.; Wiley VCH: Weinheim, Germany, 2003.

42. Adriano, D.C. Trace Elements in Terrestrial Environments. Biogeochemistry, Bioavailability and Risks of Metals; Springer: New York, NY, USA, 2001.

43. McLaughlin, M.J.; Bell, M.J.; Wright, G.C.; Cozens, G.D. Uptake and partitioning of cadmium by cultivars of peanut (Arachis hypogaea L.). Plant Soil 2000, 222, 51-58. [CrossRef]

44. Dixit, V.; Pandey, V.; Shyam, R. Different antioxidative responses to cadmium in roots and leaves of pea (Pisum sativum L. cv. Azad). J. Exp. Bot. 2001, 52, 1101-1109. [CrossRef] [PubMed]

45. Codex Alimentarius Commission. Joint FAO/WHO Food Standards Programme Codex Committee on Contaminants in Foods. In Proceedings of the FAO/WHO Food Standards Programme Fifth Session, Hague, The Netherlands, 21-25 March 2011.

46. Bose, S.; Chandraya, S.; Rai, V.; Bhattacharyya, A.K.; Ramanathan, A.L. Trannslocation of metals in pea plants grown on various amendment of electroplating industrial sludge. Bioresour. Technol. 2008, 99, 4467-4475. [CrossRef] [PubMed]

47. Eid, E.M.; Shaltout, K.H.; Alamri, S.A.M.; Sewelam, N.A.; Galal, T.M.; Brima, E.I. Prediction models for evaluating heavy metal uptake by Pisum sativum L. in soil amended with sewage sludge. J. Environ. Sci. Health Part A 2019, 55, 151-160. [CrossRef] [PubMed]

48. Radford, P.J. Growth analysis formulae-Their use and abuse. Crop Sci. 1967, 7, 171-175. [CrossRef]

49. Wilke, B.M. Determination of chemical and physical soil properties. In Manual for Soil Analysis-Monitoring and Assessing Soil Bioremediation; Margesin, R., Schinner, F., Eds.; Springer: Heidelberg, Germany, 2005; pp. 47-95.

50. Allen, S. Chemical Analysis of Ecological Materials; Blackwell Scientific Publications: London, UK, 1989.

51. Ghosh, M.; Singh, S.P. A review on phytoremediation of heavy metals and utilization of its byproducts. Appl. Ecol. Environ. Res. 2005, 3, 18. [CrossRef]

52. Kummer, A.C.B.; Filho, H.G.; Lobo, T.F.; Lima, R.A.D.S. Composted sewage sludge in replacement of mineral fertilization on wheat production and development. J. Braz. Assoc. Agric. Eng. 2016, 36, 706-714. [CrossRef]

53. Statsoft. Statistica Version 7.1.; Statsoft Inc.: Tulsa, OK, USA, 2007.

(C) 2020 by the authors. Licensee MDPI, Basel, Switzerland. This article is an open access article distributed under the terms and conditions of the Creative Commons Attribution (CC BY) license (http://creativecommons.org/licenses/by/4.0/). 